ORIGINAL ARTICLE

\title{
Benefit of antenatal glucocorticoids according to the cause of very premature birth
}

\author{
L Foix-L'Helias, O Baud, R Lenclen, M Kaminski, T Lacaze-Masmonteil
}

Arch Dis Child Fetal Neonatal Ed 2005;90:F46-F48. doi: 10.1136/adc.2003.042747

Correspondence to:
Dr Lacaze-Masmonteil,
Antoine-Béclère University
Hospital, Assistance
Publique/Hôpitaux de
Paris, 157 rue de la porte
de Trivaux, 92141
Clamart, Cedex, France;
tlacaze@club-internet.fr

Accepted 8 June 2004
In this observational study performed in a large cohort of very preterm singletons, respiratory outcome was found to be strongly dependent on the cause of premature delivery. Although less apparent in infants born to mothers with chorioamnionitis, exposure to antenatal glucocorticoids remained significantly associated with a decrease in the incidence of respiratory distress syndrome after adjustment for the main cause of premature birth.
$\mathrm{T}$ he use of antenatal glucocorticoids (AGs) in mothers at risk of preterm delivery significantly decreases the incidence of both respiratory distress syndrome (RDS) and neonatal death. ${ }^{1}$ Few studies have examined the relation between AG treatment and neonatal outcome taking into account the cause of premature delivery. ${ }^{2}$ However, this question is of importance because the incidence of several prematurity related complications, such as RDS and bronchopulmonary dysplasia (BPD), varies according to the cause of preterm birth. In the current context of knowledge, new randomised controlled trials would not be feasible, and observational studies are needed. In this multicentre cohort study, we hypothesised that the magnitude of the AG treatment outcome is influenced by the circumstance of administration.

\section{METHODS}

This retrospective study was carried out in five major perinatal centres located in the Paris area. All inborn singletons, born between 1993 and 1996, with gestational age ranging from 24 to 31 completed weeks of gestation, were included. Whereas surfactant therapy had already become the standard of care in France, the use of AGs continuously and dramatically increased over this relatively short period, from a rate of 30\% in 1993 to about 80\% in 1996.

Mothers were considered as treated if glucocorticoids were administered at any time before delivery. Infants were classified into one of the following circumstances.

- Chorioamnionitis: infants born to mothers with chorioamnionitis but without prolonged ( $>24$ hours) rupture of membranes. Chorioamnionitis was defined by the association of preterm labour and at least two of the following criteria: (a) maternal temperature greater than $38^{\circ} \mathrm{C}$; (b) maternal serum $C$ reactive protein concentration $>20 \mathrm{mg} /$ $\mathrm{l}$; (c) positive bacterial culture of amniotic fluid (amniocentesis); (d) documented early onset neonatal sepsis.

- Prolonged rupture of membranes: infants born to mothers with prolonged rupture of the membranes ( $>24$ hours), including those with chorioamnionitis.

- Pre-eclampsia: infants without intrauterine growth restriction born to mothers with pre-eclampsia.

- Intrauterine growth restriction (IUGR): infants with antenatally suspected IUGR and birth weight below the 10th centile.

- Other causes: all other, including idiopathic preterm labour.
RDS, BDP, and hospital mortality were analysed in relation to exposure to AG treatment and cause of preterm delivery. All outcomes were recorded until the babies were discharged home or died in hospital. Two BPD indicators were used: the need for supplemental oxygen at 28 days of life and 36 weeks of postmenstrual age.

\section{RESULTS}

The study cohort consisted of 670 singletons. The rates of RDS, BPD at 28 days, and hospital mortality were significantly lower in infants exposed to AGs (table 1). In the case of premature rupture of the membranes or pre-eclampsia, the use of AG was associated with a significantly lower rate of RDS. There was no significant association between the AG treatment and either respiratory morbidity or hospital mortality among infants with IUGR or in infants born to mothers with chorioamnionitis. However, in all groups except for chorioamnionitis, there was a trend in favour of lower RDS and mortality in infants born to treated mothers. For each outcome, interactions between the AG treatment and the cause of premature delivery were tested, and none were significant.

The relation between the cause of premature delivery and the AG treatment on the one hand, RDS, BPD, and death on the other hand, were estimated in a multiple logistic regression model (table 2). The risk of RDS varied with the cause of the preterm delivery. The impact of AG treatment appeared overall positive, although statistically significant only for RDS.

\section{DISCUSSION}

These results need to be discussed considering the limits of the method. Firstly, in an observational study, indication bias cannot be excluded. Several potential confounding factors were taken into account in the analyses, but there may be other unknown predictors not considered in this study. Moreover, our survey was limited to inborn babies, thus avoiding the negative effects of a postnatal transfer and possibly overestimating the impact of AG treatment. In contrast, no distinction was made between completed and uncompleted courses of AGs, and therefore the impact of the treatment may have been underestimated.

Infants born to women with histological chorioamnionitis have previously been reported to have a decreased incidence of RDS but an increased risk of eventually developing BPD,

Abbreviations: AG, antenatal glucocorticoid; BPD, bronchopulmonary dysplasia; IUGR, intrauterine growth restriction; RDS, respiratory distress syndrome 
Table 1 Neonatal outcome according to antenatal glucocorticoid treatment by cause of preterm delivery

\begin{tabular}{|c|c|c|c|c|c|c|c|c|}
\hline & \multicolumn{2}{|l|}{ RDS } & \multicolumn{2}{|c|}{ BPD at 28 days } & \multicolumn{2}{|c|}{ BPD at 36 weeks } & \multicolumn{2}{|c|}{ Hospital death } \\
\hline & $\%$ & $\mathrm{p}$ Value & $\%$ & p Value & $\%$ & p Value & $\%$ & p Value \\
\hline \multicolumn{9}{|l|}{ Total } \\
\hline Not treated $(n=285)$ & 58.2 & & 29.9 & & 11.7 & & 19.6 & \\
\hline Treated $(n=385)$ & 43.1 & 0.001 & 23.1 & 0.07 & 8.5 & 0.21 & 12.7 & 0.004 \\
\hline \multicolumn{9}{|l|}{ Chorioamnionitis } \\
\hline Not treated $(n=52)$ & 34.6 & & 29.5 & & 15.9 & & 15.4 & \\
\hline Treated $(n=45)$ & 40.0 & 0.58 & 30.0 & 0.96 & 13.2 & 0.72 & 15.6 & 0.98 \\
\hline \multicolumn{9}{|l|}{ PROM } \\
\hline Not treated $(n=61)$ & 45.9 & & 28.3 & & 8.7 & & 24.6 & \\
\hline Treated $(n=141)$ & 27.7 & 0.01 & 18.5 & 0.17 & 8.2 & 0.92 & 13.5 & 0.05 \\
\hline \multicolumn{9}{|l|}{ Pre-eclampsia } \\
\hline Not treated $(n=42)$ & 85.7 & & 27.8 & & 5.6 & & 14.3 & \\
\hline Treated $(n=40)$ & 65.0 & 0.03 & 28.9 & 0.91 & 8.3 & 0.64 & 12.5 & 0.81 \\
\hline \multicolumn{9}{|l|}{ IUGR } \\
\hline Not treated $(n=55)$ & 63.6 & & 37.8 & & 22.7 & & 21.8 & \\
\hline Treated $(n=96)$ & 51.0 & 0.13 & 26.4 & 0.18 & 10.5 & 0.06 & 12.5 & 0.13 \\
\hline \multicolumn{9}{|l|}{ Other } \\
\hline Not treated $(n=75)$ & 65.3 & & 26.7 & & 6.7 & & 20.0 & \\
\hline Treated $(n=63)$ & 54.0 & 0.17 & 19.0 & 0.32 & 3.5 & 0.44 & 9.5 & 0.09 \\
\hline
\end{tabular}

BPD, bronchopulmonary dysplasia; IUGR, intrauterine growth restriction; PROM, prolonged rupture of the membranes; RDS, respiratory distress syndrome.

Table 2 Neonatal outcome according to antenatal glucocorticoid treatment and cause of preterm delivery: multiple logistic regression

\begin{tabular}{|c|c|c|c|c|c|c|c|c|c|c|c|c|}
\hline & \multicolumn{3}{|l|}{ RDS } & \multicolumn{3}{|c|}{ BPD at 28 days } & \multicolumn{3}{|c|}{ BPD at 36 weeks } & \multicolumn{3}{|c|}{ Hospital death } \\
\hline & aOR & $95 \% \mathrm{Cl}$ & p Value & aOR & $95 \% \mathrm{Cl}$ & p Value & aOR & $95 \% \mathrm{Cl}$ & p Value & aOR & $95 \% \mathrm{Cl}$ & p Value \\
\hline \multicolumn{13}{|l|}{ Antenatal } \\
\hline glucocorticoids & & & 0.04 & & & 0.23 & & & 0.28 & & & 0.12 \\
\hline No & 1.00 & & & 1.00 & & & 1.00 & & & 1.00 & & \\
\hline Yes & 0.67 & 0.45 to 0.99 & & 0.73 & 0.43 to 1.23 & & 0.69 & 0.34 to 1.37 & & 0.66 & 0.39 to 1.12 & \\
\hline \multicolumn{13}{|l|}{ Cause of preterm } \\
\hline Chorioamnionitis & 1.00 & & & 1.00 & & & 1.00 & & & 1.00 & & 0.41 \\
\hline PROM & 0.81 & 0.47 to 1.42 & & 0.90 & 0.42 to 1.93 & & 1.13 & 0.44 to 2.92 & & 0.58 & 0.28 to 1.20 & \\
\hline Pre-eclampsia & 7.16 & 3.85 to 13.3 & & 1.91 & 0.88 to 4.12 & & 0.80 & 0.25 to 2.49 & & 0.76 & 0.35 to 1.68 & \\
\hline IUGR & 3.13 & 1.96 to 5.01 & & 2.81 & 1.50 to 5.32 & & 2.86 & 1.27 to 6.46 & & 1.17 & 0.63 to 2.16 & \\
\hline Other & 3.25 & 2.00 to 5.30 & & 1.28 & 0.64 to 2.55 & & 0.59 & 0.21 to 1.72 & & 0.80 & 0.42 to 1.54 & \\
\hline
\end{tabular}

The multiple logistic regression includes antenatal glucocorticoid treatment, cause of preterm delivery, gestational age, sex, year, and centre.

BPD, bronchopulmonary dysplasia; IUGR, intrauterine growth restriction; PROM, prolonged rupture of the membranes; RDS, respiratory distress syndrome; aOR, adjusted odds ratio.

compared with infants born to mothers without chorioamnionitis. ${ }^{3}$ Although antenatal infection or inflammation may have a beneficial short term effect on lung maturation, partially through the modulating effects of endotoxin and proinflammatory cytokines on surfactant synthesis, the mechanisms underlying the relations between fetal exposure to inflammation and the potential development of BPD remain to be determined. Our data also confirm the previously established relation between IUGR and BPD at 36 weeks. ${ }^{5}$

Our study suggests that the beneficial impact of AG treatment on the prevention of RDS and neonatal death is present, irrespective of the main cause of preterm delivery. However, the benefit of AGs on lung maturation was less apparent in infants born to mothers with chorioamnionitis, the cause of preterm delivery associated with the lowest risk of RDS. This less apparent benefit in the case of chorioamnionitis is supported by recent experimental data suggesting that antenatal exposure to endotoxin results in better lung maturation than does maternal administration of glucocorticoids, but interferes with the ability of the lung to downregulate the inflammatory reaction. ${ }^{6}$ However, this observation should not call into question the use of AGs in mothers with chorioamnionitis. Indeed, very premature babies born in this context are at high risk of developing either intraventricular haemorrhage or periventricular leucomalacia, and AGs are highly effective in reducing the incidence of these complications in this population. ${ }^{7}$

\section{ACKNOWLEDGEMENTS}

We are grateful to René Frydman, Michel Dehan, Dominique Cabrol, Guy Moriette, Michel Tournaire, Christophe Dupont, Yves Ville, Alain Paupe, and Philippe Narcy, the respective directors of the divisions of Obstetrics and Neonatology in the following hospitals: AntoineBéclère University hospital (AP-HP), Cochin Port-Royal University hospital (AP-HP), Saint-Vincent de Paul University hospital (AP-HP), Poissy regional hospital, and Saint-Germain en Laye regional hospital. We thank Kristi Watterberg, MD, for her valuable input into the interpretation of the results.

\section{Authors' affiliations}

L Foix-L'Helias, O Baud, T Lacaze-Masmonteil, Division of Neonatology, Antoine-Béclère University Hospital, Assistance Publique/ Hôpitaux de Paris, Clamart, France

R Lenclen, Division of Neonatology, Poissy-Saint Germain Hospital, Poissy, France

L Foix-L'Helias, M Kaminski, Epidemiological Research Unit on Perinatal and Women's Health, INSERM U149, Villejuif, France

\section{REFERENCES}

1 Crowley P. Prophylactic corticosteroids for preterm birth. Cochrane Database Syst Rev 2000;(2):CD000065.

2 Elimian A, Verma U, Canterino J, et al. Effectiveness of antenatal steroids in obstetric subgroups. Obstet Gynecol 1999;93:174-9.

3 Baud O, Zupan V, Lacaze-Masmonteil T, et al. The relationships between antenatal management, the cause of delivery and neonatal outcome in a large cohort of very preterm infants. Br J Obstet Gyncol 2000;107:877-84. 
4 Watterberg KL, Demers LM, Scott SM, et al. Chorioamnionitis and early lung inflammation in infants in whom bronchopulmonary dysplasia develops. Pediatrics 1996;97:210-15.

5 Egreteau L, Pauchard JY, Semama DS, et al. Chronic oxygen dependency in infants born at less than 32 week's gestation. Incidence and risk factors. Pediatrics 2001; 108:e16.
6 Jobe AH. Antenatal factors and the development of bronchopulmonary dysplasia. Semin Neonat 2003;8:9-17.

7 Baud O, Foix-L'Helias L, Kaminski M, et al. Antenatal

glucocorticoids treatment and cystic periventricular leukomalacia

in very preterm infants. N Engl J Med 1999;341.

1190-6.

\section{IMAGES IN NEONATAL MEDICINE}

doi: $10.1136 /$ adc. 2003.048140

Long line complication: accidental cannulation of ascending lumbar vein

A

long line was inserted at the medial aspect of the left knee of a 27 week gestation neonate. The line used was Vygon Premicath, 27 gauge, inserted up to $10 \mathrm{~cm}$. A test radiograph with contrast showed the dye flowing upwards at the left side of the vertebrae in the anatomical position of the left ascending lumbar vein. Thus it appears that the long line had gone into this small tributary of the left common iliac vein instead of going along the vein and entering the inferior vena cava. This highlights the value of using contrast to check the long line position.

A De, A Imam Neonatal Unit, Luton and Dunstable Hospital, Luton, UK; ambasyam@yahoo.co.uk

Competing interests: none declared

\section{REFERENCE}

1 Reece A, Ubhi T, Craig AR, et al. Positioning long lines: contrast versus plain radiography. Arch Dis Child Fetal Neonatal Ed 2002:87:F233.
Figure 1 Radiograph with contrast showing dye flowing upwards at the left side of the vertebrae in the anatomical position of the left ascending lumbar vein.

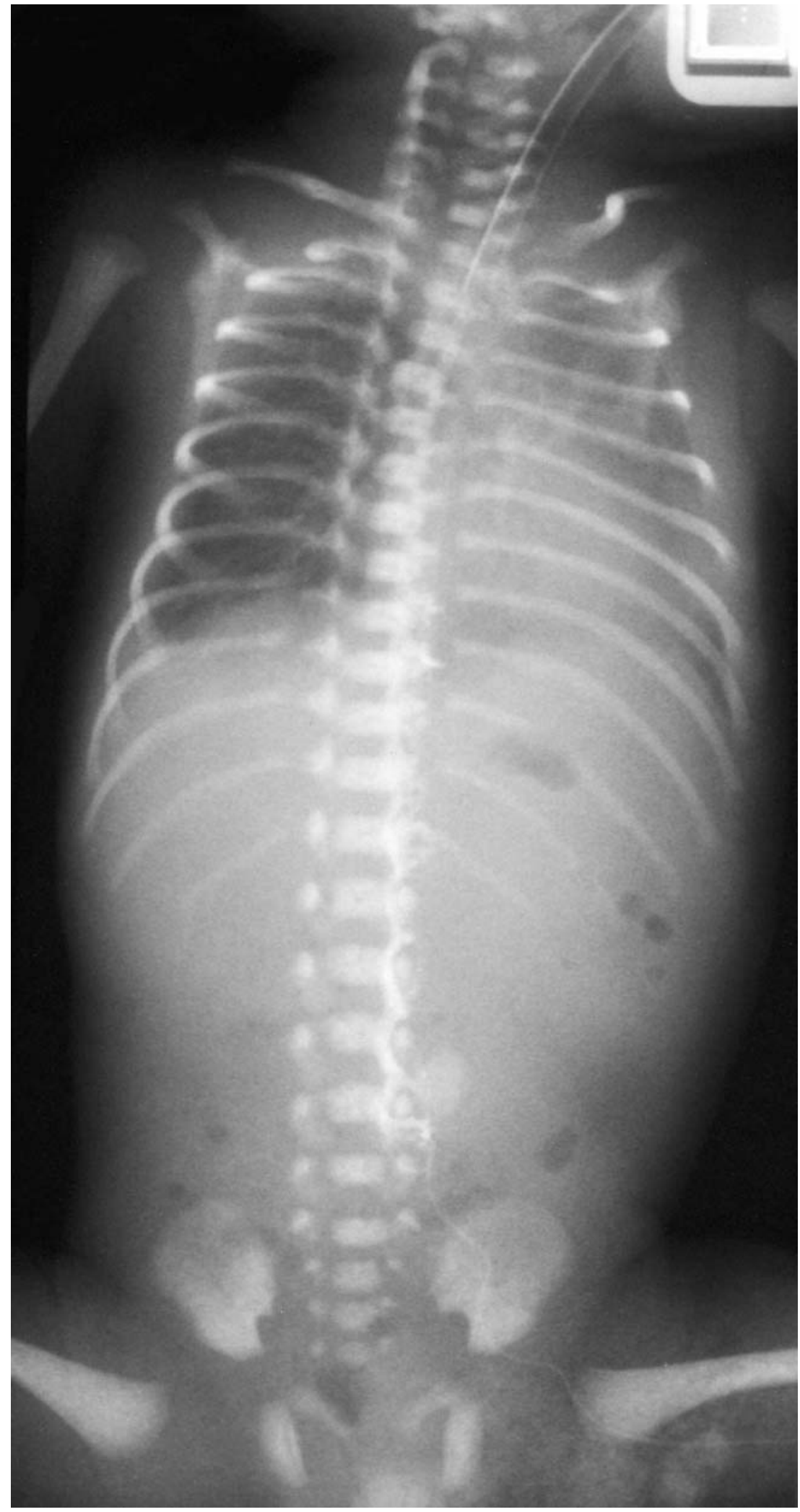

